\title{
ADVANCES IN PROCESSING AND INTERPRETING MAGNETIC GRADIOMETER DATA FOR UXO DETECTION
}

\author{
Ben Spurgeon', Troy Wilson ${ }^{1}$, Ole G. Engels ${ }^{2}$ and Christian Barthel ${ }^{3}$ \\ ${ }^{1}$ Geosoft Europe Limited, Pangbourne, Reading, UK \\ ${ }^{2}$ HarbourDom Consulting GmbH, Cologne, Germany \\ ${ }^{3}$ Barthel \& Schreiber GmbH, Duisburg, Germany
}

\section{INTRODUCTION}

Geophysical mapping techniques offer a cost-effective solution to clean up and reclaim land contaminated with Un-eXploded Ordnance (UXO). Modern survey techniques involving rapid-sampling gradiometers generate large, high resolution land and marine datasets which need to be properly processed by UXO detection and removal companies to extract the maximum amount of information possible.

Data Processing and Analysis (DPA) software packages such as UX-Detect ${ }^{\mathrm{TM}}$ can rapidly process and interpret large volumes of magnetic or EM data. By using two-dimensional Fast Fourier Transforms (2D-FFT), geophysical filters and inversion routines it is possible to identify UXO found in clusters, detect deeper targets and provide reliable depth and location information for planning remediation programs.

\section{THEORY}

Magnetic anomalies are difficult to interpret for a number of reasons. To begin with, the total magnetisation of buried ferrous objects is the resultant of two vectors, an induced component caused by the geomagnetic field aligning magnetic dipoles within the body, and a remanent component, often called the permanent magnetisation, which is 'frozen' into the body.

In most man-made ferromagnetic bodies, the remanent magnetisation dominates. This explains, in part, many E-W orientated magnetic anomalies seen in site investigation surveys and test sites where man-made objects are found. However, ordnance items which have seen a ground impact tend to have their magnetic history (i.e. remanent magnetisation) erased - the magnetic dipoles within the ordnance being randomly rearranged as the impact energy dissipates through the body (Altshuler, 1996). The shape of the body also effects the magnetic signature of buried UXO. Altshuler has shown that extended ordnance items are more easily magnetised along their semi-major axis. In addition, extended ferromagnetic bodies which lie at some angle to the Earth's geomagnetic field tend to pull the total magnetisation vector into the semi-major axis. These factors add to the complexity of magnetic UXO anomalies.

One solution to this problem is to use the analytic signal $|\mathrm{A}(\mathrm{x}, \mathrm{y})|$ or energy envelope to obtain first order approximations for the locations of buried UXO within a survey area. The analytic 
signal uses any three orthogonal components of the total field grid to derive a value for the magnitude of the total magnetisation vector. For simplicity, the two horizontal derivatives can be calculated from the total field gridded data $(d T / d x, d T / d y)$ and combined with the vertical derivative data $(d T / d z)$ recorded during the survey, according to the equation

$$
|A(x, y)|=\sqrt{\left(\frac{d T}{d x}\right)^{2}+\left(\frac{d T}{d y}\right)^{2}+\left(\frac{d T}{d z}\right)^{2}}
$$

where $T$ represents the total magnetic field. The amplitude of the peaks in the analytic signal grid are related to the magnetic moment of the UXO and are located directly above the poles of the anomalous body.

Euler's Homogeneity equation is commonly applied to gridded potential field data to derive depth and locations of anomalous magnetic and gravity sources (Reid et al., 1990). For each target, Euler's equation can be solved for multiple positions within a window centered on the target location. The equation takes the form

$$
\left(x-x_{0}\right) \frac{\partial T}{\partial x}+\left(y-y_{0}\right) \frac{\partial T}{\partial y}+\left(z-z_{0}\right) \frac{\partial T}{\partial z}=N(B-T)
$$

where $\left(x_{0}, y_{0}, z_{0}\right)$ is the position of a magnetic source whose total field $T$ is detected at $(x, y, z)$. The remaining parameters describe the regional value of the total field, denoted by $B$, and the Structural Index of the causative body, $N$, which is a measure of the decay of the magnetic field with distance.

The method does not make any assumptions of the physical parameters of the body causing the anomaly or its source of magnetisation - this information is contained within the Structural Index. The avoidance of assumptions is a strength of the technique over other depth inversion routines which often omit the effects of remanent magnetisation and/or assume point dipole sources.

\section{DATA ACQUISITION}

Modern magnetic surveys acquire enormous amounts of digital data at very high sampling densities. A number of recent gradiometer surveys have deployed arrays of up to seven instruments, spaced at intervals ranging from $25-100 \mathrm{~cm}$ and recording data every $5-20 \mathrm{~cm}$ along line. The lower sensors are typically positioned $10-30 \mathrm{~cm}$ above the ground and the upper sensor a further $50-70 \mathrm{~cm}$ above this, depending on the expected target depths and the field conditions. At this sort of sampling density, a typical UXO survey of dimensions $100 \mathrm{~m}$ $\times 100 \mathrm{~m}$ will record data at 300,000 individual locations. Including sample locations, which are recorded simultaneously using Differential GPS, the resultant data file will be around $10 \mathrm{Mb}$ in size when stored in ASCII format.

Traditional methods of interpreting this data, such as interpreting line profiles by hand, cannot effectively process such large datasets typically containing many hundreds of magnetic anomalies. There is a need for a semi-automated, software solution to the problem to process and interpret magnetic UXO data rapidly and effectively. A number of geophysical solutions are available commercially and we present here some results from part of a large marine survey processed using one such system. 


\section{CASE STUDY}

In our example UXO gradiometer data was acquired using a GPS-positioned towed-array system consisting of three vertical gradiometers positioned $70 \mathrm{~cm}$ apart. The array was towed underwater in an inland river (the line paths being shown in Figure 1) at a nominal clearance of $55 \mathrm{~cm}$ above the river bed. The gradiometer data was imported into the OASIS database to handle the volume of data and facilitate quality control of the survey. This allowed the processor to examine the data for field acquisition problems such as spikes, step functions, etc. which needed to be reviewed and corrected before the data could be leveled for interpretation. The amount of leveling required was a function of how well the gradiometers were

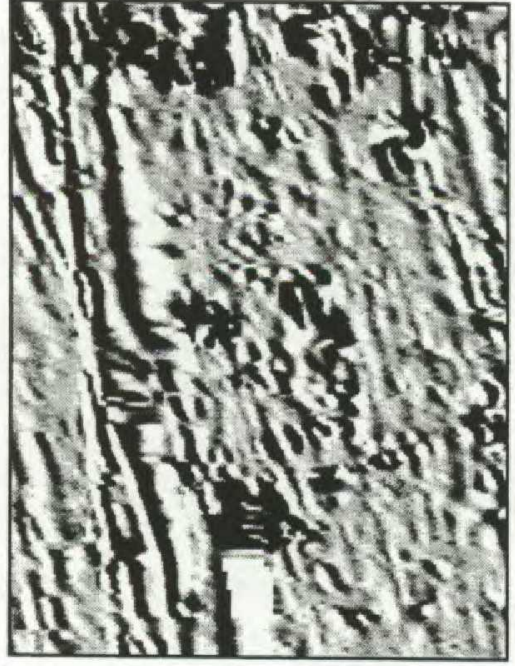

Figure 2: Unleveled vertical gradient grid showing line-to-line base level problems. calibrated before and during the survey (Figure 2). The vertical gradient data was corrected for $\mathrm{n}^{\text {th }}$ order polynomial level shifts by applying geophysical filters. Long wavelength (regional) signal was calculated and stored as a separate

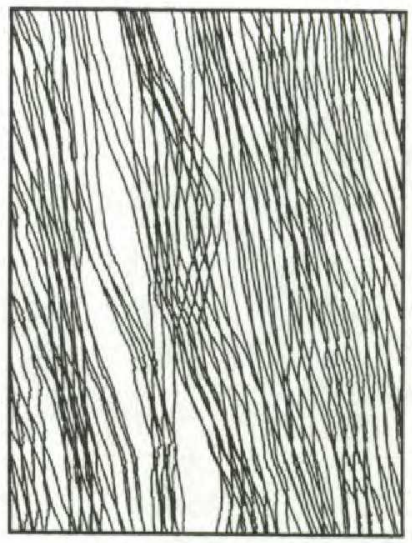

Figure 1: Survey line paths for 3-sensor array. channel within the database. It was then examined by the data processing specialist to ensure that no real anomalies were present in the signal. The regional field was then subtracted from the original data to produce a leveled data channel. The data was then gridded (Figure 3) using a minimum curvature algorithm to prepare it for use in the analytic signal calculation of the UX-Detect ${ }^{\mathrm{TM}}$ system. Lag was not present in these data so no corrections were applied prior to the gridding operation.

To calculate the analytic signal, a two-dimensional Fast Fourier Transform (2D-FFT) routine was used to derive a grid of the total magnetic field from the gradiometer data. A convolution (smoothing) filter was applied to the vertical gradient grid before running the 2D-FFT routine to reduce ringing effects due to steep gradients associated with near-surface UXO. Horizontal derivative grids were then derived from the total field grid using $3 \times 3$ convolution filters. The analytic signal grid was generated by combining the three derivative grids $(d T / d x$, $d T / d y, d T / d z$ ) according to equation (1). The analytic signal grid was used as input into an automated peak picking algorithm such as that developed by Blakely (Blakely \& Simpson, 1986) to find and isolate potential UXO targets. The target locations were written into a new database and used to position the Euler window for calculating depths.

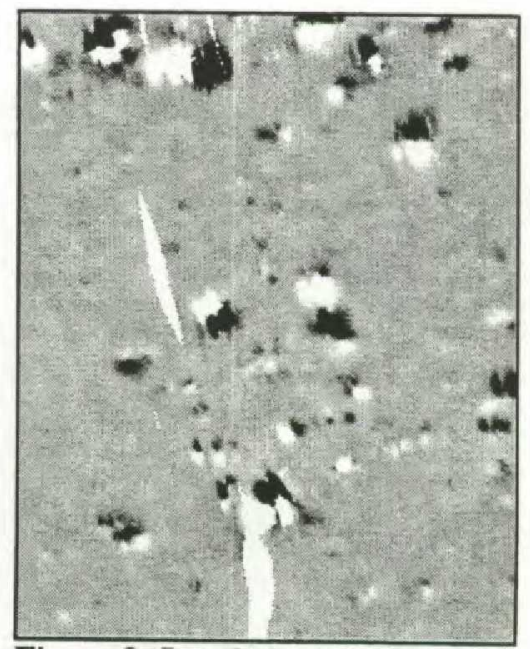

Figure 3: Leveled vertical gradient grid. Individual anomalies due to buried UXO are now clearly seen. 


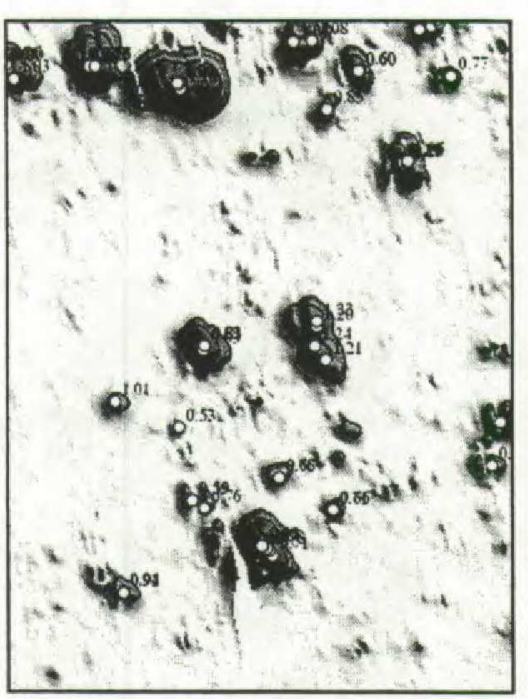

Figure 4: The analytic signal grid showing calculated depths and locations of targets.

Depths and locations of all magnetic targets were derived by solving Euler's Homogeneity equation for targets isolated using the peaks of the analytic signal grid. Window size and Structural Index were adjusted as necessary, for example, where clustering of magnetic UXO or extended objects were identified by the magnetic field grids. Application of statistical analysis including scatter plots of depth solutions against percentage errors to the depth was used to remove poor solutions before the final interpretation.

Interactive visual links between the original data, the depth and target solutions, and the magnetic field grids assisted the interpreter when distinguishing between real UXO targets and man-made artifacts. The final database and interpretation maps (Figure 4) together provide a complete and accurate repository of information about target location and depth for all magnetic targets within the survey area.

\section{CONCLUSION}

Modern geophysical survey techniques have resulted in an order of magnitude increase in the volume of data acquired in the field. In no other area have we seen a larger increase than in the use of magnetic and electromagnetic methods in UXO detection surveys. Traditional processing techniques (such as interpreting line profiles by hand) have in the past proved to be ineffective for handling large datasets which has lead to poor interpretation of UXO contaminated sites. These datasets require modern processing tools to properly database, process, interpret and present results which provide more accurate information for UXO detection and removal companies. The experience and knowledge base of the interpreter is enhanced when provided with this accurate information, leading to improved target selection.

\section{ACKNOWLEDGEMENT}

The authors would like to thank Barthel \& Schreiber GmbH for their permission to use the UXO data presented in this paper and also for their insight in developing UXO survey techniques.

\section{REFERENCES}

Altshuler, T.W., 1996, Shape and orientation effects on magnetic signature prediction for unexploded ordnance, UXO forum (March $26^{\text {th }}-28^{\text {th }}$ ), Williamsberg, Virginia, pp282-291.

Blakely, R.J. \& Simpson, R.W., 1986, Approximating edges of source bodies from magnetic or gravity anomalies, Geophysics, 51, 1494-1498.

Reid, A.B., Allsop, J.M., Granser, H., Millett, A.J. \& Somerton, I.W., 1990, Magnetic interpretation in three dimensions using Euler deconvolution, Geophysics, 55, 80-91. 